\title{
Ian Snook Prizes 2015
}

\section{Ian Snook Prize 2015}

The 2015 Ian Snook Prize has been awarded to Puneet Kumar Patra for his original and thoughtful work, Two-Dimensional Time-Reversible Ergodic Maps with Provisions for Dissipation [1]. Patra responded to the problem posed for the 2015 Prize with a family of two-dimensional time-reversible mappings of the unit square where $q$ is a coordinate and $p$ is a momentum:

$$
(q, p) \stackrel{M}{\rightarrow}\left(q^{\prime}, p^{\prime}\right) ;\left(q^{\prime},-p^{\prime}\right) \stackrel{M}{\rightarrow}(q,-p) .
$$

His mappings run the gamut from uniform ergodic coverage of the square through dissipative fractal strange attractors to limit cycles. These maps parallel developments in nonequilibrium statistical physics with continuous equations of motion. His work is pedagogical and useful to an understanding of irreversibility and the Second Law of Thermodynamics. Both Loschmidt's and Zermélo's paradoxes have analogs in Patra's maps. We first became acquainted with Puneet through his work with his Professor Baidurya Bhattacharya (Indian Institute of Technology, Kharagpur) which we refereed for the 2014 Journal of Chemical Physics [2].

We congratulate Puneet and wish him well on his career with many more contributions to our understanding of the world around us.

[1] P.K. Patra, Two-Dimensional Time-Reversible Ergodic Maps with Provisions for Dissipation, CMST 22, 61-70 (2016).

[2] P.K. Patra, B. Bhattacharya, A Deterministic Thermostat for Controlling Temperature Using All Degrees of Freedom, J. Chem. Phys. 140, 064106 (2014).

William Graham Hoover and Carol Griswold Hoover Ruby Valley, Nevada, USA

\section{Ian Snook Additional Prize 2015}

The 2015 Ian Snook Additional Prize has been won by Puneet Kumar Patra. His winning paper is published in this volume of the CMST [1]. We congratulate the Winner.

[1] P.K. Patra, Two-Dimensional Time-Reversible Ergodic Maps with Provisions for Dissipation, CMST 22, 61-70 (2016). 